\title{
On Superior Reliability of Effective Signal Power versus RSSI in LoRaWAN
}

\author{
Ahmed Abdelghany, Bernard Uguen, Christophe Moy, Senior Member, IEEE, Dominique Lemur \\ Univ Rennes, CNRS, IETR - UMR 6164 \\ F-35000, Rennes, France \\ \{ahmed.abdelghany, bernard.uguen, christophe.moy, dominique.lemur\}@univ-rennes1.fr
}

\begin{abstract}
With the growth of the Internet of Things (IoT), it is critical to understand the packet transmission performance in Low Power Wide Area Network (LPWAN), especially LoRaWAN. Previous works on LoRaWAN network performance analysis, either simulation-based or field measurement, are considering the Packet Delivery Rate (PDR) as a major measure of the network performance and its dependency on the signal impairments. However, the Effective Signal Power (ESP) and its huge variability along with the different frequency bands, for each location, are not generally considered. In this paper, an in-depth investigation of the frequency dependency of the PDR is done by performing an outdoor measurement campaign in the area of the Campus Beaulieu in Rennes. From each different location, the ESP and SINR (Signal-to-Interference-plus-Noise Ratio) values are obtained as well as the influence of these parameters on the PDR is evaluated at each frequency band independently. In the given results, the feasibility of using the ESP is proven by its enlarged range when the SINR is very low, unlike the RSSI which has a limitation. This investigation manifests and gives important guidelines for using ESP in the future IoT applications.
\end{abstract}

Index Terms-IoT, LPWAN, LoRa, Packet Delivery Rate, RSSI, Effective Signal Power, Onsite Measurement, Smart City, Channel Reciprocity

\section{INTRODUCTION}

Internet of Things (IoT) has been employed in a wide range of fields, such as health monitoring, livestock, smart city, etc [1]. Many IoT applications require a low-cost deployment network with the characteristics of large coverage and low power consumption, which is difficult to be fulfilled using the conventional cellular or the short-range wireless networks. For providing this connectivity, Low Power Wide Area Network (LPWAN) is considered the leading technology, especially the LoRaWAN whose design compromises between low energy consumption and a large communication range. This ability is mostly exhibited in the typical LoRa devices, which can cover distances of more than $10 \mathrm{~km}$ using a maximum transmit power of $14 \mathrm{dBm}$ in the unlicensed ISM spectrum while maintaining extremely long battery lifetimes at the scale of up to several years [2].

LoRaWAN is an open standard network protocol, i.e. managed and updated by the LoRa Alliance [3]. LoRaWAN utilizes LoRa as its modulation schema. For the performance of the entire LoRaWAN network, the transmission quality of data packets has a significant impact on it [4]. Thus, the reliability of communications in the network is impaired by the channel quality of the link between the end node and the gateway. Consequently, packet loss may occur due to the channel attenuation or interference. This transmission failure affects various IoT applications and even causes serious outcomes. Furthermore, the data integrity and accuracy in IoT data analytics may be reduced by a large amount of packet loss. Therefore, LoRaWAN supports both unconfirmed and confirmed messaging. Confirmed messaging is most probably used for important sensor data. In contrary to the unconfirmed message, the end node requires the message to be acknowledged as received by the network server when sending a confirmed message. Consequently, the end node will retransmit the data packet when it does not receive the acknowledgment. However, this retransmission requires an extra energy consumption that impacts the battery life of end node devices plus occupying an additional spectrum, raising the interfering level and occupying time that could be used for uplinks.

Recently, many papers are addressing the different origins of LoRa packet loss. For example, in [5] the authors conduct experiments to evaluate a characterization of LoRaWAN frame collision conditions. Moreover, the Packet Delivery Rate (PDR) and coverage of LoRa technology are evaluated for outdoor cases in [6] and [7]. Based on empirical results in [8], path loss models are developed for LoRaWAN communications and compared with widely used empirical models. In [9], authors study the impact of environmental factors on the performance of LoRa, and show that higher temperatures decrease the Received Signal Strength Indicator (RSSI) and may drastically affect PDR. While in [10], the first attempt to investigate the PDR in a real city-scale LoRaWAN network is presented. However, these previous works do not evaluate the Effective Signal Power (ESP), i.e. introduced in [5], as one of the main factors affecting the PDR rather than using RSSI. Besides, they do not focus on the frequency dependency of PDR.

In this paper, an in-depth investigation of the PDR using the trace collected from a real network deployment in the area of the Campus Beaulieu in Rennes. From various end node locations, the parameters that have a direct impact on PDR are extracted and analyzed per each frequency band. Hence, the whole ESP values in the experiment are extensively 
evaluated, and the other possible causes of packet loss are thoroughly explained.

The remainder of this document is organized as follows. Section II presents the measurement overview. Section III provides sufficient detail about the derivation of ESP. The key factors of PDR are then presented and commented in Section IV. Hence, Section V provides an evaluation of the PDR's frequency dependency. Finally, the work is concluded in Section VI.

\section{System and Measurement Setup}

The main aim of the proposed experiment is obtaining the SINR, and received power of the uplink and downlink, against the PDR for each channel. This is done by transmitting confirmed LoRa packets sequentially at the typical frequency bands for Europe, i.e 8 channels with center frequency $f_{k} \in\{867.1,867.3,867.5,867.7,867.9,868.1,868.3,868.5\}$ $\mathrm{MHz}$, and $125 \mathrm{kHz}$ bandwidth $W$. As shown in Figure 1, an IoT node is placed at various locations in two different areas. This end node is implemented using a Pycom card, i.e. programmed in the MicroPython language, composed of an Expansion Board and a LoPy 4 module which can support LoRa wireless connectivity [11], as shown in Figure 2c. While a Tektelic KONA Macro Gateway is used whose antenna is fixed on the roof of the university building [12], as shown in Figure 2a and $2 b$.

First, the Pycom node transmits an uplink packet for each specific channel $f_{k}$. While the gateway attempts to send an acknowledgment by default at the same frequency as the message transmitted. Consequently, the node writes the information of the last received downlink packet (packet number, ESP, etc.) to the payload of the next uplink packet. For this experiment, a desktop computer runs a Python program is used as an Application Server (AS) which receives data from the LoRa Network Server (LNS), as well as LoRa metadata with all parameters of the LoRaWAN transmission $\left(f_{k}\right.$, Spreading Factor (SF), $W$, RSSI, SINR, etc.). Those data are processed as explained in the following sections. They are provided to the research community on this online repository [13].

\section{EFfective Signal Power}

The LoRa system can operate at SNR (Signal-to-noise ratio) below $0 \mathrm{~dB}$ as LoRa signals can be decoded with signal power below the noise floor [5]. Based on the independence between signal and noise, the measured power $P_{r}$ at the input RF chain is the sum of the signal power $P_{s}$, the potential interference power $P_{i}$ and the noise power $P_{n}$. These are related on a linear scale as $P_{r}=P_{s}+P_{i}+P_{n}$ and the signal to interference plus noise ratio is:

$$
\rho=\frac{P_{s}}{P_{i}+P_{n}}
$$

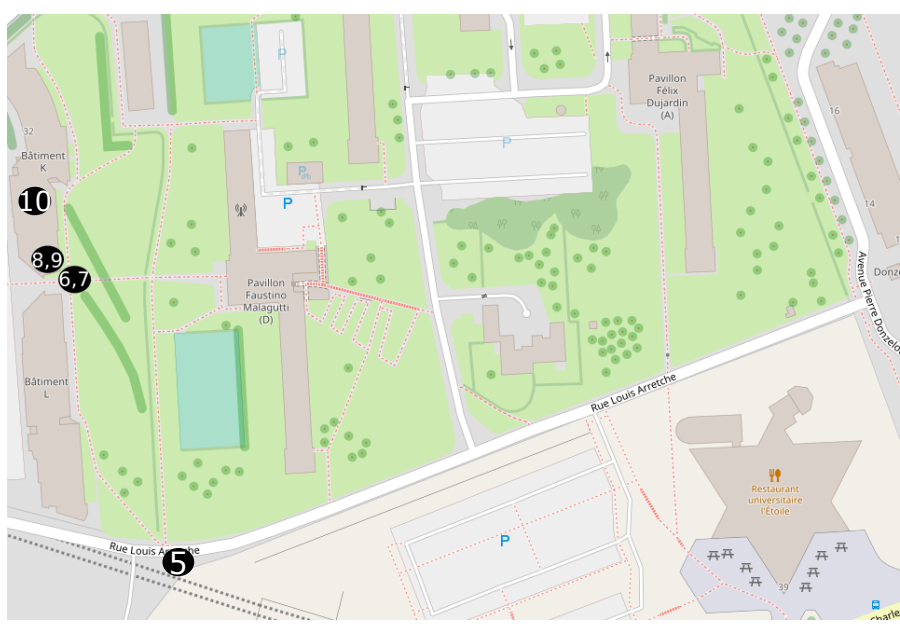

(a) The end node locations in the area of the Residence University Beaulieu. Location 8, 9 and 10 are indoor.

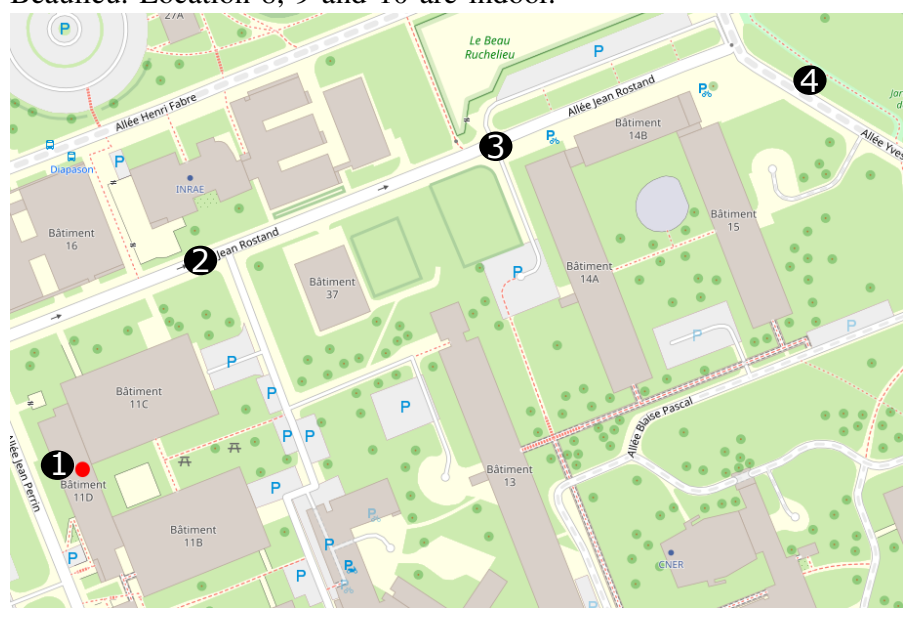

(b) The end node locations in the area of the Campus Beaulieu. Location 1 is indoor.

Fig. 1: Positions of the node and the gateway are labeled by black, and red markers respectively. (Cby OpenStreetMap Contributers)

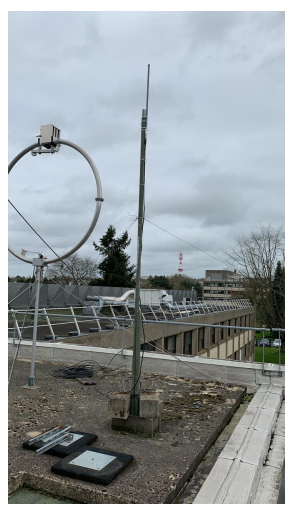

(a) Position of the gateway monopole (b) The gateway con fixed on the nected to the antenna rod and connected to roof. cable. a battery at location 7 .

Fig. 2: Views from the end node and gateway sites. 
By assuming the power quantities are expressed in milliwatt, these can be redefined on a logarithmic scale as:

$$
\begin{aligned}
& R S S I_{d B m}=10 \log _{10} P_{r}, \\
& E S P_{d B m}=10 \log _{10} P_{s},
\end{aligned}
$$

and the SINR is:

$$
S I N R_{d B}=10 \log _{10} \rho .
$$

Hence, the computation of the RSSI follows as:

$$
\begin{aligned}
R S S I_{d B m} & =10 \log _{10}\left(P_{s}+\frac{P_{s}}{\rho}\right) \\
& =10 \log _{10}\left(P_{s}\right)+10 \log _{10}\left(1+\frac{1}{\rho}\right) \\
& =E S P_{d B m}+10 \log _{10}(1+\rho)-10 \log _{10}(\rho) \\
& =E S P_{d B m}+10 \log _{10}\left(1+10 \frac{S I N R_{d B}}{10}\right)-S I N R_{d B},
\end{aligned}
$$

then the ESP can be expressed as:

$$
E S P_{d B m}=R S S I_{d B m}+S I N R_{d B}-10 \log _{10}\left(1+10^{0.1 S I N R_{d B}}\right) .
$$

By ignoring the potential interference power $P_{i}$, the RSSI can also be defined with respect to the thermal noise power $P_{n}$ as:

$$
\begin{aligned}
R S S I_{d B m} & =10 \log _{10}\left(P_{n} \rho+P_{n}\right) \\
& =10 \log _{10}\left(P_{n}\right)+10 \log _{10}(\rho+1) \\
& =10 \log _{10}\left(P_{n}\right)+10 \log _{10}\left(1+10^{0.1 S I N R_{d B}}\right)
\end{aligned}
$$

with

$$
P_{n}=k_{B} T W \cdot 1000
$$

where the Boltzmann constant $k_{B}$ is $1.381 \times 10^{-23} \mathrm{~J} \mathrm{~K}^{-1}$ and the temperature $T$ is set to $293.15 \mathrm{~K}$. By using equation 6 , the ESP can also be defined as:

$$
E S P_{d B m}=10 \log _{10}\left(P_{n}\right)+S I N R_{d B} .
$$

At this point, these can be estimated as:

$$
R S S I_{d B m} \approx-123+10 \log _{10}\left(1+10^{0.1 S I N R_{d B}}\right)
$$

and

$$
E S P_{d B m} \approx-123+S I N R_{d B}
$$

Taking the measured data in Rennes as an example, Figure 3 shows a comparison between RSSI and ESP against different SINR values. For positive SINR, ESP coincides with RSSI, but it differs for the negative SINR. The most striking observation is that the raw measurement of RSSI and ESP provided by the experimental setup are very precisely limited by expressions 10 and 11, while those lower limits are obtained by only considering the thermal noise assumption. Thus, the RSSI distribution saturates when the received power is approaching
$-120 \mathrm{dBm}$. In contrary to RSSI, ESP at negative SINR goes below this previous limited value of the RSSI. It is worth mentioning that when the ESP limitation gets to an extreme value, it refers to the maximum receiving sensitivity, i.e. $-142 \mathrm{dBm}$ as mentioned in the Tektelic gateway specifications sheet [12]. Since some packets are received with SINR below $0 \mathrm{~dB}$ in the practical experiments, ESP is more reliable to be used for PDR analysis. It could be utilized for IoT localization also, particularly ESP instead of RSSI fingerprinting.

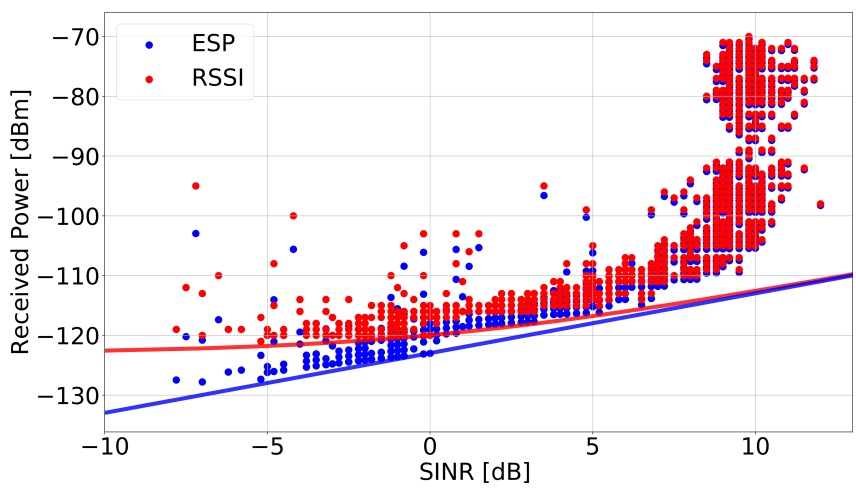

Fig. 3: Comparison of the measured RSSI and ESP against SINR.

\section{KEY FACTORS OF PDR}

To check the plausibility of utilizing the ESP, the main causes of the packet loss events are discussed in this section. Two main aspects can affect the PDR, which are measured received power and SINR, as detailed in the following subsections.

\section{A. Impact of received power}

Figure 4a explores how downlink PDR changes along with uplink RSSI and ESP. The result shows that high PDR values reduce significantly with the decrease of RSSI, which is similar when the ESP decreases. However, RSSI distribution is limited when approaching the aforementioned noise floor limitation, i.e. $R S S I \approx-120 \mathrm{dBm}$ at $S I N R=0 \mathrm{~dB}$. One can observe the rather large gap between the ESP values and the RSSI values, especially when the received power is below $-120 \mathrm{dBm}$. Therefore, ESP has a simpler and more natural relationship with PDR than RSSI.

\section{B. Impact of SINR}

The result shows a general rise in PDR when the colorcoded ESP or SINR increases, as shown in Figure 4b. This direct proportionality is established in the previous works [10]. On the other hand, it can be noticed that this long-term observation exhibits two kinds of unexpected behavior. These should be related in two manners in how the interference can affect the relationship between received power and SINR. The first unexpected situation is to have at the same time a low SINR and a rather high received power as depicted in Figure 3. 
Those situations correspond to a case where the SINR is probably measuring an additional interfering power coming from a colliding packet. The second unexpected situation is having a high SINR and nevertheless, a low packet success rate as depicted in Figure $4 \mathrm{~b}$, which is most probably due to a typically accomplished interference in the downlink. Those observations suggest that the joint observation of SINR and RSSI or ESP can be exploited for detecting interfered conditions.

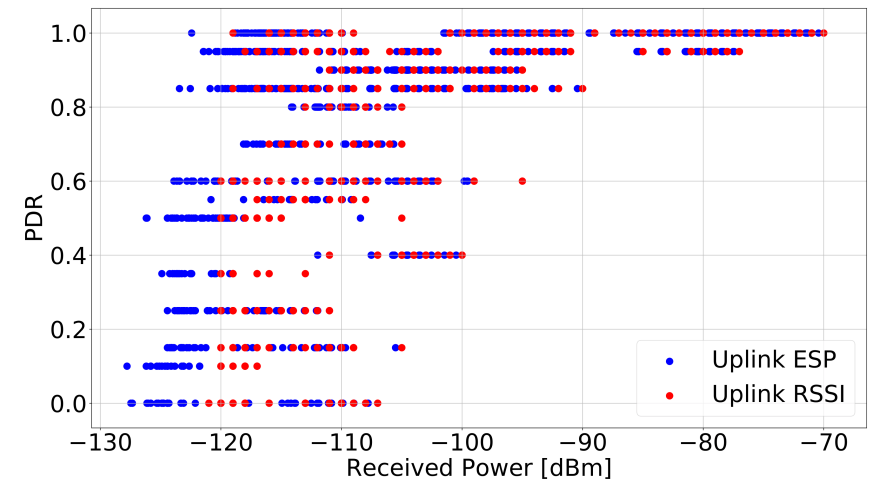

(a) Changes with the measured received power.

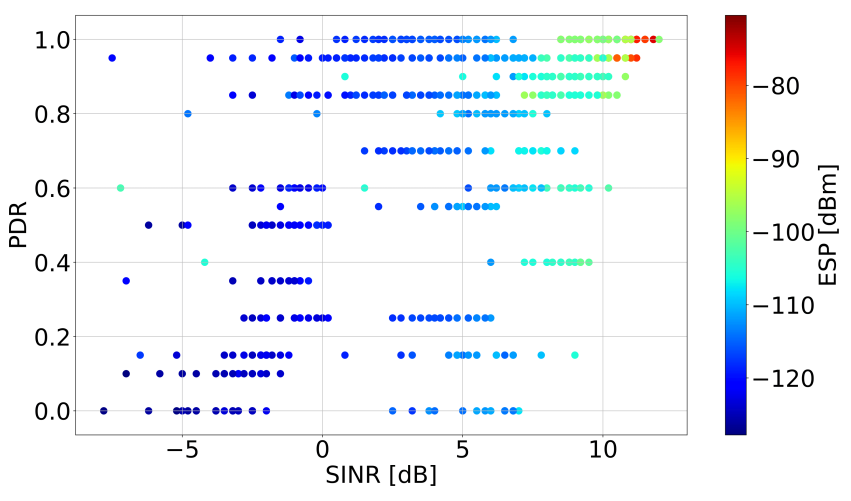

(b) Changes with the measured SINR.

Fig. 4: PDR for various received power and SINR values.

\section{FREQUENCY DEPENDENCY OF PDR}

The radio channel between a gateway and an IoT node in a LoRaWAN is very specific at each location. Its quality has an impact on the capability to establish a successful transmission, as it is confirmed in the previous sections. Moreover, the LoRa channel with such a small bandwidth of $125 \mathrm{kHz}$ is almost flat. However, this section shows that there are significant variations of the channel gain over different LoRa center frequencies $f_{k}$, with only a $200 \mathrm{kHz}$ spacing in the $868 \mathrm{MHz}$ ISM band. Consequently, the impact of the channel on the successful packet rate is investigated for each center frequency independently. As an example shown in Figure 5a, the absolute Channel State Information (CSI) from location 4 is frequency selective with a deep fade of more than $10 \mathrm{~dB}$ depth. On the other hand, channel reciprocity is clearly manifested between the uplink and downlink CSI apart from a constant value whose compensation would require an accurate calibration of the transceiver RF chains. This reciprocity feature could be utilized for the physical layer security between the node and the gateway in future IoT applications. While the PDR values preserve almost the same magnitude ranks across all the frequency bands, as shown in Figure 5b. Downlink PDR values are here preferred, because they are more correlated with the absolute CSI shape in these near transmission conditions.

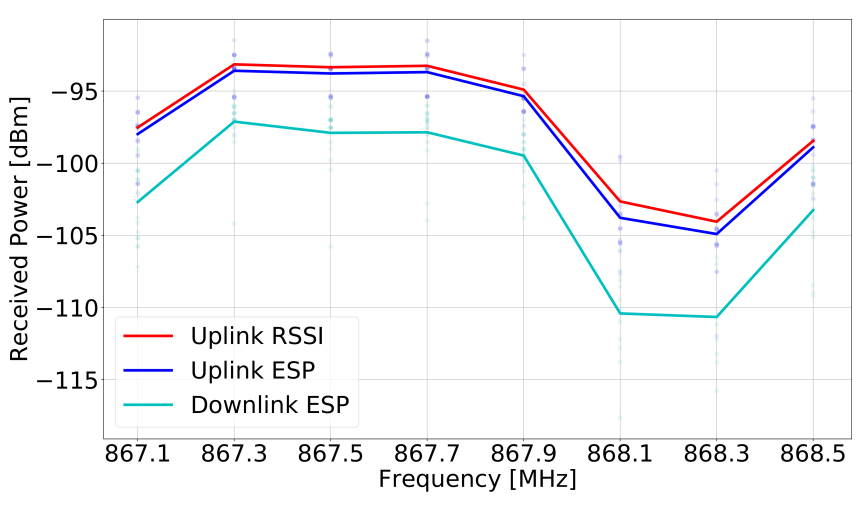

(a) ESP values.

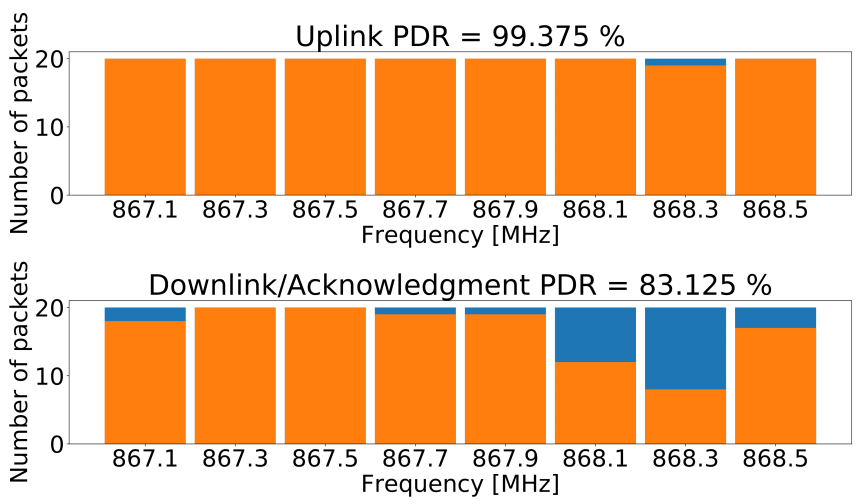

(b) PDR values.

Fig. 5: Result from location 4 at each different frequency band.

\section{A. Channel shape variability over the ISM-Band}

Figure 6a shows exemplary signal level spectra observed in all the scenarios of the experiment, averaged over the whole trial. A progressive reduction is observed in the received power across location 1 to 10 . This reduction is reasonable as the distance between the gateway and the node gradually increases from $\approx 5 \mathrm{~m}$ at location 1 to $\approx 760 \mathrm{~m}$ at location 10 . The first category of CSI is represented in location $1,3,8,9$, and 10 which shows moderate fades with a low indication of multipath propagation. Thus, the variation of signal level is less than $6 \mathrm{~dB}$ over the $1.5 \mathrm{MHz}$ frequency range. The opposite is the signal level spectrum of location 2, 4, 5, 6, and 7 which stand for another category of channels showing strong frequency selectivity. This category indicates multipath propagation with 
a path delay so it has many deep fades with up to $15 \mathrm{~dB}$ depth, in such a small bandwidth of $1.5 \mathrm{MHz}$ only.

\section{B. Impact of the channel on PDR}

The PDR values on each center frequency for all the locations are shown in Figure 6b. Location 1 and 3 show total successful transmissions across the whole frequency bands which is reasonable as they already have almost flat fading channels, and high received power as shown in Figure 6a. While PDR values in the other locations preserve the same pattern of their CSI shape. For example, location 5 has PDR values that fit well with respective properties of the corresponding frequency bands, particularly at $867.1 \mathrm{MHz}$ and 867.3 MHz with low PDR values like their ESP values. This confirms the strong frequency dependency of the PDR in the LoRa system.

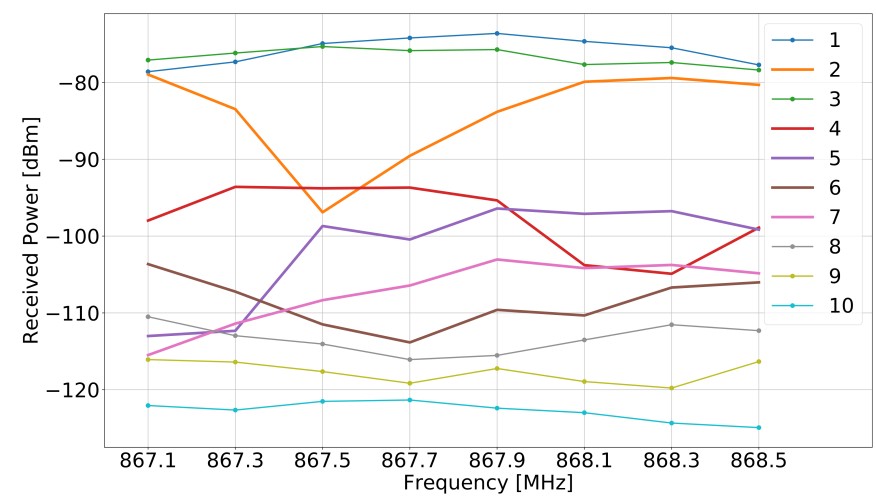

(a) ESP values over different frequency bands at each location.

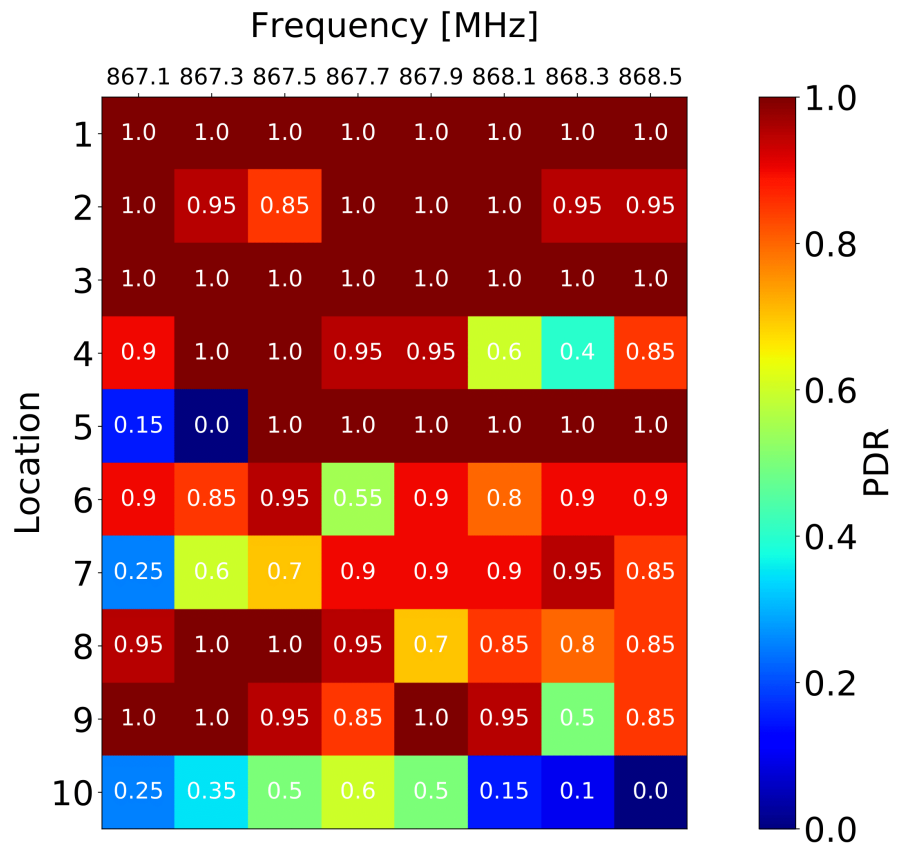

(b) PDR values over different frequency bands at each location.

Fig. 6: Result from the ten different locations.

\section{Conclusion}

This paper presents the feasibility of utilizing the ESP rather than RSSI in many prospective IoT applications. Besides, the frequency dependency of PDR in LoRa is studied. Thus, a measurement campaign is carried out in the city of Rennes to estimate the channel parameters (RSSI, ESP, SINR) from different node locations. Hence, PDR is investigated against ESP at each different frequency band. Consequently, the presented data indicate that ESP has a more natural relationship with PDR than RSSI. This result demonstrates that ESP is more reliable as it overcomes the RSSI limitation, especially at low SINR $(<0 \mathrm{~dB})$.

For future work, this paper highly recommends using ESP for the potential IoT applications, especially localization using ESP fingerprinting. Thus, the different channel gains can be used as additional information to increase the localization accuracy. On the other hand, this frequency dependency of ESP value should be utilized to improve PDR.

\section{ACKNOWLEDGMENT}

We are grateful to Jean François Legendre from Gwagenn company for borrowing us the gateway [14].

\section{REFERENCES}

[1] A. A. Ghany, B. Uguen and D. Lemur, "A Pre-processing Algorithm Utilizing a Paired CRLB for TDoA Based IoT Positioning," 2020 IEEE 91st Vehicular Technology Conference (VTC2020Spring), Antwerp, Belgium, 2020, pp. 1-5, doi: 10.1109/VTC2020Spring48590.2020.9128385.

[2] R. Oliveira, L. Guardalben and S. Sargento, "Long range communications in urban and rural environments," 2017 IEEE Symposium on Computers and Communications (ISCC), Heraklion, 2017, pp. 810-817, doi: 10.1109/ISCC.2017.8024627.

[3] LoRa-Alliance - https://www.lora-alliance.org/

[4] Augustin, A.; Yi, J.; Clausen, T.; Townsley, W.M. A Study of LoRa: Long Range \& Low Power Networks for the Internet of Things. Sensors 2016, 16, 1466.

[5] Andri Rahmadhani and Fernando Kuipers. 2017. Understanding collisions in a LoRaWAN

[6] L. Li, J. Ren and Q. Zhu, "On the application of LoRa LPWAN technology in Sailing Monitoring System," 2017 13th Annual Conference on Wireless On-demand Network Systems and Services (WONS), Jackson, WY, 2017, pp. 77-80, doi: 10.1109/WONS.2017.7888762.

[7] S. Wang et al., "Performance of LoRa-Based IoT Applications on Campus," 2017 IEEE 86th Vehicular Technology Conference (VTCFall), Toronto, ON, 2017, pp. 1-6, doi: 10.1109/VTCFall.2017.8288154.

[8] R. El Chall, S. Lahoud and M. El Helou, "LoRaWAN Network: Radio Propagation Models and Performance Evaluation in Various Environments in Lebanon," in IEEE Internet of Things Journal, vol. 6, no. 2, pp. 2366-2378, April 2019, doi: 10.1109/JIOT.2019.2906838.

[9] Cattani, M.; Boano, C.A.; Römer, K. An Experimental Evaluation of the Reliability of LoRa Long-Range Low-Power Wireless Communication. J. Sens. Actuator Netw. 2017, 6, 7.

[10] Q. Liu, Y. Mu, J. Zhao, J. Feng and B. Wang, "Characterizing Packet Loss in City-Scale LoRaWAN Deployment: Analysis and Implications," 2020 IFIP Networking Conference (Networking), Paris, France, 2020, pp. 704-712.

[11] Pycom documentation: https://GitHub.com/PyCom/PyCom-libraries

[12] Tektelic KONA Macro IoT Gateway. https://www.tektelic.com/uploads/Brochures/Kona\%20Macro.pdf

[13] Measurement Data. https://gitlab.com/ahmednagy/lorawan-beaulieumeasurement-2020

[14] Gwagenn company website. http://www.gwagenn.com/en/home/ 